\title{
Review
}

\section{Early Gross Motor Milestones in Duchenne Muscular Dystrophy}

\author{
Giulia Norcia ${ }^{\mathrm{a}}$, Simona Lucibello ${ }^{\mathrm{a}, \mathrm{b}}$, Giorgia Coratti ${ }^{\mathrm{a}, \mathrm{b}}$, Roberta Onesimo $^{\mathrm{c}}$, Elisa Pede $^{\mathrm{b}}$, \\ Gloria Ferrantini $^{\mathrm{a}, \mathrm{b}}$, Claudia Brogna ${ }^{\mathrm{b}}$, Graziamaria Cicala ${ }^{\mathrm{b}}$, Sara Carnicella ${ }^{\mathrm{a}}$, Nicola Forcina ${ }^{\mathrm{a}}$, \\ Lavinia Fanelli ${ }^{\mathrm{a}}$, Marika Pane ${ }^{\mathrm{a}, \mathrm{b}, 1}$ and Eugenio Mercuri ${ }^{\mathrm{a}, \mathrm{b}, 1, *}$ \\ ${ }^{a}$ Centro Clinico Nemo, Fondazione Policlinico Universitario Agostino Gemelli IRCCS, Rome, Italy \\ ${ }^{\mathrm{b}}$ Pediatric Neurology, Università Cattolica del Sacro Cuore, Rome, Italy \\ ${ }^{\mathrm{c}}$ Institute of Pediatrics, Fondazione Policlinico Universitario Agostino Gemelli IRCCS, Rome, Italy
}

Pre-press 26 April 2021

\begin{abstract}
.
Background: Over the last few years there has been increasing attention to detect early signs of impairment in young Duchenne muscular dystrophy boys but less has been reported on whether the delay may also affect the very early aspects of motor development, such as gross motor milestones.

Objective: The aim of this study was to retrospectively assess the age when early motor milestones were achieved in Duchenne muscular dystrophy.

Methods: The study is a retrospective analysis of data collected as part of a larger natural history project. Information on past medical history, collected at the time the boys were seen for the first time, were recorded and re available on clinical notes and on electronic CRF.

Results: Data were collected in 134 DMD boys. Sitting was achieved at 7.04 months. The \% of DMD boys not achieving sitting by 9.4 months was 10\%, ranging from $2 \%$ in the boys with mutations before exon 44 to $33 \%$ in those beyond exon 63 . Walking was achieved at a mean age of 16.35 months. The \% of DMD boys not achieving independent walking by 18 months was $17 \%$, ranging from $9 \%$ in the boys with mutations between 44 and 51 to $42 \%$ in those beyond exon 63 .

Conclusions: Our results showed that the risk of a delay in sitting and walking was increasingly high in patients with mutations predictive of the involvement of different brain dystrophin isoforms.
\end{abstract}

Keywords: Duchenne muscular dystrophy, early motor milestones, dystrophin isoforms, gross motor milestones

\section{INTRODUCTION}

Duchenne muscular dystrophy is a X linked disorder with mutations in the Xp21 gene resulting in reduced production of dystrophin [1]. The diagnosis of DMD is still often achieved after the age of 4 years

\footnotetext{
${ }^{1} \mathrm{MP}, \mathrm{EM}$ are both senior authors.

${ }^{*}$ Correspondence to: Eugenio Mercuri, Largo Agostino Gemelli 8, 00152, Rome, Italy. E-mail: eugeniomaria.mercuri@uni catt.it.
}

[1-5] but the onset of clinical signs is often around the age of 2 to 3 years, at the time when boys fail to achieve the ability to walk fast, run and jump/hop. Over the last few years there has been increasing attention to detect early signs of impairment in young DMD boys [6-11]. In patients who had early diagnosis the use of developmental scales such as Griffiths [8] and Bayley's [6, 7] allowed to identify lower performances compared to their peers, affecting not only gross motor function but also other aspects of 
development such as language or visual perceptual abilities. These findings indicate that the delay may be at least also partly due to the involvement of the dystrophin isoforms in the brain. This was also suggested by the fact that lower scores were more often found in patients with mutations (downstream exon 44) which are associated with additional involvement of two dystrophin isoforms, Dp140 and Dp71, expressed in brain, with consequences for brain function [8].

Less has been reported on whether the delay may also affect the very early aspects of motor development that are typically achieved in the first year of life, such as sitting and walking.

The aim of this study was to retrospectively assess the age when early motor milestones were achieved and a possible correlation with the site of mutation and the involvement of different brain dystrophin isoforms.

\section{MATERIALS AND METHODS}

The study is a retrospective analysis of data collected as part of a larger natural history project. As part of this project, information on past history, collected at the time the boys were seen for the first time, are recorded and re available on clinical notes and on electronic CRF. The study was approved by the Ethics committee of our institution.

As the information collected is part of the clinical routine, consent to anonymously record the data in a database was obtained by the parents for the underage boys. As part of the study, we collected the information from all the patients with a genetically confirmed diagnosis of DMD seen in our clinics. Details on the mutations were noted.

When interviewed, families were asked to report the age when their boy had achieved sitting and walking independently. Sitting was defined as the ability to sit independently without any support and walking was defined as the ability to walk for more than a few steps without holding to furniture or people. As this information was collected retrospectively, we could not obtain more specific information, such as walking 10 meters, that could have been collected in a prospective study. In order to establish whether this modality of data collection was appropriate, we used a control group of typically developing boys in the same age range of our study cohort, asking their families to report the age of sitting and walking independently in their children.

\section{Statistical analysis}

The data were compared to the WHO normative data for milestones, identifying the number of patients falling outside the $95 \%$ confidence interval of the WHO data [12].

Clinical data were also compared to the site of mutations and the subsequent involvement of different isoforms identifying different subgroups: mutations located upstream from exon 44 that preserve Dp140 and Dp71. In patients mutations placed between intron 44 and exon 51 the effect on Dp140 expression is hard to predict and is defined as of uncertain significance.

In contrast, the involvement of Dp140 is certain to those with mutations after exon 51. Patients with mutations located on the 3' side, from exon 63 are considered to affect all dystrophin isoforms, including not only Dp140 but also Dp71.

Variables were described by mean and standard deviations. Mann-Whitney U test was used to compare mean age of motor milestones acquisitions between DMD cohort and typically developing control group; Kruskal-Wallis test was used to compare the DMD subgroups according the site of mutation. Version 23 of the SPSS software (SPSS, Inc.) was used for all statistical analyses, setting the significance at $p<0.05$.

\section{RESULTS}

Data were collected in 134 DMD boys (age range 1.09-32.58) by interviewing the parents. Forty-five of the $134(33.6 \%)$ had mutation before exon 44 , therefore preserving both Dp140 and Dp71; 43 (32.1\%) had mutations between 44 and 50 defined as of uncertain significance in relation to the involvement of Dp140; 40 (29.9\%) after exon 51, with involvement of Dp140 and the remaining $6(4.5 \%)$ beyond exon 63, affecting all dystrophin isoforms, including both Dp140 and Dp71.

The interview was also conducted in 150 typically developing boys of similar age range.

\section{Sitting}

\section{DMD cohort}

Sitting independently was achieved at a mean age of 7.04 months in the whole DMD cohort. The percentage of boys achieving sitting by 5.9 months (50th percentile according to WHO) was $2.98 \%$ in the whole DMD cohort. Mean age at achievement 
Table 1

Sitting in DMD and typically developing control group

\begin{tabular}{lcccccc}
\hline & $\begin{array}{c}\text { Typically developing } \\
\text { control group (n:150) }\end{array}$ & $\begin{array}{c}\text { All DMD } \\
\text { (n:134) }\end{array}$ & $\begin{array}{c}<44 \\
(\mathrm{n}: 45)\end{array}$ & $\begin{array}{c}44-50 \\
(\mathrm{n}: 45)\end{array}$ & $\begin{array}{c}51-62 \\
(\mathrm{n}: 40)\end{array}$ & $\begin{array}{c}\geq 63 \\
(\mathrm{n}: 6)\end{array}$ \\
\hline Mean (SD) & $7.07(1.04)$ & $7.04(1.78)$ & $6.58(1.31)$ & $6.96(1.65)$ & $7.52(2.14)$ & $7.91(2.49)$ \\
Min-max & $5-9$ & $4-13.5$ & $4-13$ & $5-12$ & $5-13.5$ & $6-12$ \\
Patient out of WHO range & $0 / 150(0 \%)$ & $13 / 134(10 \%)$ & $1 / 45(2 \%)$ & $4 / 43(9 \%)$ & $6 / 40(15 \%)$ & $2 / 6(33 \%)$ \\
\hline
\end{tabular}

Table 2

Walking in DMD and typically developing control group

\begin{tabular}{|c|c|c|c|c|c|c|}
\hline & $\begin{array}{l}\text { Typically developing } \\
\text { control group (n:150) }\end{array}$ & $\begin{array}{l}\text { All DMD } \\
(\mathrm{n}: 142)\end{array}$ & $\begin{array}{l}<44 \\
(\mathrm{n}: 50)\end{array}$ & $\begin{array}{l}44-50 \\
(\mathrm{n}: 42)\end{array}$ & $\begin{array}{l}51-62 \\
(\mathrm{n}: 43)\end{array}$ & $\begin{array}{l}\geq 63 \\
(\mathrm{n}: 7)\end{array}$ \\
\hline Mean (SD) & $12.26(2.11)$ & $16.35(5.13)$ & $15.77(4.50)$ & $15.19(2.91)$ & $17.45(6.43)$ & $20.85(7.88)$ \\
\hline Min-max & $8-18$ & $10-48$ & $11-30$ & $10-24$ & $12-48$ & $12-36$ \\
\hline Patient out of WHO range & $0 / 150(0 \%)$ & 24/142 (17\%) & $7 / 50(14 \%)$ & $4 / 42(9 \%)$ & $10 / 43(23 \%)$ & $3 / 7(42 \%)$ \\
\hline
\end{tabular}

values ranged between 6.58 in the boys with mutations before exon 44 to 7.91 in those with mutations beyond exon 63 . The difference between the different mutations subgroups was not significant ( $p=0.229)$.

The percentage of boys not achieving sitting by 9.4 months (outside 95\% confidence interval according to WHO) was $10 \%$ in the whole DMD cohort with values ranging from $2 \%$ in the boys with mutations before exon 44 to $33 \%$ in those with mutations beyond exon 63. (Table 1)

\section{Typically developing control group}

Sitting independently was achieved at a mean age of 7.07 months in the control group.

The difference between DMD and typically developing boys was not significant $(p=0.339)$

\section{Walking}

\section{DMD cohort}

Walking independently was achieved at a mean age of 16.35 months in the whole DMD cohort. The percentage of boys achieving walking by 12.0 months (50th percentile according to WHO) was $23.88 \%$ in the whole DMD cohort. Mean age at achievement values ranged with mean values ranging between 15.19 in the boys with mutations between 44 and 51 to 20.85 in those with mutations beyond exon 63 . The difference between the different mutation subgroups was not significant $(p=0.88)$.

The percentage of DMD boys not achieving independent walking by 18 months (outside $95 \%$ confidence interval according to WHO) was $17 \%$ in the whole DMD cohort with values ranging between $9 \%$ in the boys with mutations between 44 and 51 to $42 \%$ in those with mutations beyond exon 63. (Table 2)

\section{Typically developing control group}

Walking independently was achieved at a mean age of 12.26 months in the control group.

The percentage of typically developing boys not achieving independent walking by 18 months (outside $95 \%$ confidence interval according to WHO) was 0 .

The difference between DMD and typically developing boys was significant $(p<0.001)$.

\section{DISCUSSION}

Over the last few years several papers have reported early clinical signs in young DMD boys (6-11), in some cases suggesting that they may be related to the involvement of the dystrophin expressed in the brain [8]. Our results suggest that to a certain extent this may also apply to very early aspects of development that typically occur in the first year. This was more obvious for the achievement of walking than for sitting. There was no difference in sitting between DMD and control group as only $10 \%$ of the overall group of DMD boys achieved independent sitting outside the range provided by the WHO. It is of interest however that, when we analyzed possible differences among DMD subgroups subdivided according to site of mutation and subsequent involvement of different brain dystrophin isoforms, the number of patients with delayed sitting was progressively higher in patients with mutations affecting different brain dystrophin isoforms, reaching 33\% in the subgroups with involvement of both Dp140 and Dp71.

The difference between DMD and control groups in the ability to walk independently was significant. This was at least partly driven by the impairment 
of dystrophin isoforms as there was a progressive increase of the number of patients with delayed walking in the subgroups of patients with mutations affecting different brain dystrophin isoforms, reaching $42 \%$ in those with involvement of both Dp140 and Dp71.

One of the limitations of our study is that the data was retrospectively collected by interview but as the diagnosis is still reached on an average of over 4 years, this was the only possible modality of data collection. The use of a control group allowed to reduce a possible bias related to this modality as the results in the control group were concordant with the WHO windows of achievements. Our results suggest that the involvement of brain dystrophin isoforms may be responsible for early signs of neurodevelopmental delay form the first year of life. As a number of patients is identified very early in life, either accidentally [4], or because of prenatal screenings or previous familial cases, these may provide the opportunity to follow these patients prospectively and define a profile of development in patients with different mutations. This will also be important as the early identification of a possible delay will help to provide early intervention.

\section{ACKNOWLEDGMENTS}

None.

\section{FUNDING}

This research did not receive any specific grant from funding agencies in the public, commercial, or not-for-profit sectors.

\section{CONFLICT OF INTEREST}

EM: Advisory Board and PI for Sarepta, Santhera, PTC, Roche, Wave, Lilly, NS; MP: Advisory Board for Sarepta, Santhera, PTC; the other authors report no disclosures discolsures.

\section{REFERENCES}

[1] Mercuri E, Bonnemann CG, Muntoni F. Muscular dystrophies. Lancet. 2019;394(10213):2025-38.

[2] Counterman KJ, Furlong P, Wang RT, Martin AS. Delays in diagnosis of Duchenne muscular dystrophy: An evaluation of genotypic and sociodemographic factors. Muscle Nerve. 2020;61(1):36-43.

[3] Birnkrant DJ, Bushby K, Bann CM, Apkon SD, Blackwell A, Brumbaugh D, et al. Diagnosis and management of Duchenne muscular dystrophy, part 1: Diagnosis, and neuromuscular, rehabilitation, endocrine, and gastrointestinal and nutritional management. Lancet Neurol. 2018;17(3): 251-67.

[4] D'Amico A, Catteruccia M, Baranello G, Politano L, Govoni A, Previtali SC, et al. Diagnosis of Duchenne Muscular Dystrophy in Italy in the last decade: Critical issues and areas for improvements. Neuromuscul Disord. 2017;27(5): 447-51.

[5] Ciafaloni E, Fox DJ, Pandya S, Westfield CP, Puzhankara S, Romitti PA, et al. Delayed diagnosis in duchenne muscular dystrophy: Data from the Muscular Dystrophy Surveillance, Tracking, and Research Network (MD STARnet). J Pediatr. 2009;155(3):380-5.

[6] Connolly AM, Florence JM, Cradock MM, Eagle M, Flanigan KM, McDonald CM, et al. One year outcome of boys with Duchenne muscular dystrophy using the Bayley-III scales of infant and toddler development. Pediatr Neurol. 2014;50(6):557-63.

[7] Connolly AM, Florence JM, Cradock MM, Malkus EC, Schierbecker JR, Siener CA, et al. Motor and cognitive assessment of infants and young boys with Duchenne Muscular Dystrophy: Results from the Muscular Dystrophy Association DMD Clinical Research Network. Neuromuscul Disord. 2013;23(7):529-39.

[8] Pane M, Scalise R, Berardinelli A, D’Angelo G, Ricotti $\mathrm{V}$, Alfieri P, et al. Early neurodevelopmental assessment in Duchenne muscular dystrophy. Neuromuscul Disord. 2013;23(6):451-5.

[9] Coratti G, Brogna C, Norcia G, Ricotti V, Abbott L, D'Amico A, et al. Longitudinal natural history in young boys with Duchenne muscular dystrophy. Neuromuscul Disord. 2019;29(11):857-62.

[10] Mercuri E, Coratti G, Messina S, Ricotti V, Baranello G, D'Amico A, et al. Revised North Star Ambulatory Assessment for Young Boys with Duchenne Muscular Dystrophy. PLoS One. 2016;11(8):e0160195.

[11] Miller NF, Alfano LN, Iammarino MA, Connolly AM, Moore-Clingenpeel M, Powers BR, et al. Natural History of Steroid-Treated Young Boys With Duchenne Muscular Dystrophy Using the NSAA, 100m, and Timed Functional Tests. Pediatr Neurol. 2020;113:15-20.

[12] Group WHOMGRS. WHO Motor Development Study: Windows of achievement for six gross motor development milestones. Acta Paediatr Suppl. 2006;450:86-95. 